\title{
Crescimento de Aegla itacolomiensis (Crustacea, Decapoda) em um arroio da Mata Atlântica no sul do Brasil
}

\author{
Raoní da Silva-Gonçalves, Georgina Bond-Buckup \& Ludwig Buckup
}

Laboratório de Carcinologia, Depto de Zoologia, PPG Biologia Animal, Universidade Federal do Rio Grande do Sul, Av. Bento Gonçalves, 9500, prédio 43435, Porto Alegre, RS, Brasil. (raoni_goncalves@yahoo.com.br)

\begin{abstract}
The growth of Aegla itacolomiensis (Crustacea, Decapoda) in an Atlantic forest stream in southern Brazil. To give more information about ecology and biology of Aegla itacolomiensis Bond-Buckup \& Buckup, 1994 was analized its growth in the nature. Monthly samples were made from June 2005 to May 2006 in Solitária Alta stream, tributary of Rio dos Sinos basin, district of Igrejinha, RS, Brazil (29 $33^{\prime} 10,2^{\prime \prime} S$; $\left.50^{\circ} 50^{\prime} 57^{\prime \prime} \mathrm{W}\right)$. The specimens were collected by dip net, sexed and after the cephalotorax measured, the animals were brought back to the stream. The growth estimated by cephalotorax length for males and females of A.itacolomiensis was analized by absolut distributional size-classes frequency. The modal frequency of cephalotorax lenght was calculated. The growth was estimated trought the modal progression. The growth curves in cefalotorax length for males and females are described respectively by the equations: $\mathrm{C}_{\mathrm{t}}=23.21\left[1-\mathrm{e}^{-0.0094(t+7.03)}\right]$ e $\mathrm{C}_{\mathrm{t}}=19.49\left[1-\mathrm{e}^{-0.0065(\mathrm{t}+11.16)}\right]$. Were found juveniles in the spring and summer. The males attained larger sizes and a higher growth rate than females. The estimated longevity was 2.2 years for males and 2.5 years for females.
\end{abstract}

KEYWORDS. Aegla itacolomiensis, growth, Crustacea, Aeglidae.

RESUMO. Analisou-se o crescimento de Aegla itacolomiensis Bond-Buckup \& Buckup, 1994, em ambiente natural, a partir de amostragens mensais, realizadas entre junho de 2005 e maio de 2006, no arroio Solitária Alta, tributário da bacia hidrográfica do Rio dos Sinos, município de Igrejinha, RS, Brasil (29³3'10,2”S; 5050'57’'W). Os indivíduos foram coletados com o auxílio de um puçá, triados por sexo, medidos quanto ao comprimento do cefalotórax (CC) e devolvidos ao arroio no mesmo local de onde foram retirados. O crescimento em comprimento de machos e fêmeas de A. itacolomiensis foi avaliado pela análise das distribuições de frequências absolutas em intervalos de classe do CC. As modas dos histogramas de frequências do CC foram verificadas. O crescimento dos indivíduos foi estimado por meio da análise da progressão das modas calculadas, resultando nas curvas de crescimento para machos e fêmeas, respectivamente: $C_{t}=23,21\left[1-\mathrm{e}^{-0,0094(\mathrm{t}+7,03)}\right]$ e $\mathrm{C}_{t}=19,49\left[1-\mathrm{e}^{-0,0065(\mathrm{t}+11,16)}\right]$. O recrutamento ocorreu na primavera e no verão. Os machos atingiram tamanhos maiores do que as fêmeas e apresentaram maior taxa de crescimento que estas. A longevidade estimada para os machos foi de 2,2 anos enquanto que para as fêmeas foi de 2,5 anos. O padrão de crescimento de A. itacolomiensis é semelhante ao de outras espécies de eglídeos anteriormente estudados.

PALAVRAS-CHAVE. Aegla itacolomiensis, crescimento, Crustacea, Aeglidae.

Os caranguejos anomuros do gênero Aegla Leach, 1820, despontam como os únicos representantes vivos da infraordem Anomura que habitam águas continentais, diferenciando-se evolutivamente de outras 1.400 espécies de anomuros de vida exclusivamente marinha, lagunar ou estuarina (Bond-BuckuP \& BucKuP, 1994). Trata-se de crustáceos neotropicais endêmicos das bacias hidrográficas da América do Sul meridional (BondBucKuP, 2003), em regiões que se encontram sob domínio dos climas temperado e subtropical.

Os eglídeos compõem, com outros macroinvertebrados, as comunidades zoobentônicas dos ambientes límnicos onde vivem, seja em rios, riachos, lagos e cavernas de águas correntes e bem oxigenadas (BOND-Buckup, 2003). Esses crustáceos são considerados elos importantes em diversas relações tróficas do seu ecossistema, servindo de alimento para rãs, peixes salmonídeos (Arenas, 1976) e aves. Alimentam-se de detritos vegetais e de larvas aquáticas de insetos (BUENo \& BOND-BUCKUP, 2004), atuando, assim, como protagonistas na dinâmica de nutrientes e no fluxo de energia dos ambientes onde vivem (EsTEVES, 1988).

O estudo do crescimento dos eglídeos em ambiente natural teve início em 1945, com trabalhos de caráter biométrico realizados por VAZ-FERREIRA et al. (1945) com Aegla uruguaiana Schmitt, 1942, seguidos por
BAHAMONDE \& López (1961), que investigaram o crescimento de A. laevis laevis (Latreille, 1818). LÓPEZ (1965) caracterizou o crescimento de A. paulensis Schmitt, 1942 e RodRIGUEZ \& HEBLING (1978) estudaram aspectos do crescimento de A. perobae Hebling \& Rodriguez, 1977. Mais recentemente, Bueno et al. (2000), Swiech-Ayoub \& MASUNARI (2001), Noro \& Buckup (2003), Boss et al. (2006) e Silva-Castiglioni et al. (2006) pesquisaram respectivamente o crescimento de A. platensis Schmitt, 1942, A. castro Schmitt, 1942, A. leptodactyla Buckup \& Rossi, 1977, A. jarai Bond-Buckup \& Buckup, 1994 e A. longirostri Bond-Buckup \& Buckup, 1994.

O objetivo deste trabalho é caracterizar os principais parâmetros do crescimento de Aegla itacolomiensis Bond-Buckup \& Buckup, 1994, bem como compará-los com os resultados obtidos com outras espécies de eglídeos.

\section{MATERIAL E MÉTODOS}

Foram realizadas amostragens mensais durante o período de doze meses, compreendidos entre junho de 2005 e maio de 2006 no arroio Solitária Alta, tributário da bacia hidrográfica do rio dos Sinos, situado no município de Igrejinha no estado do Rio Grande do Sul, Brasil (29³3’10,2”S; 50 50'57’W) (Fig. 1). O arroio apresenta- 


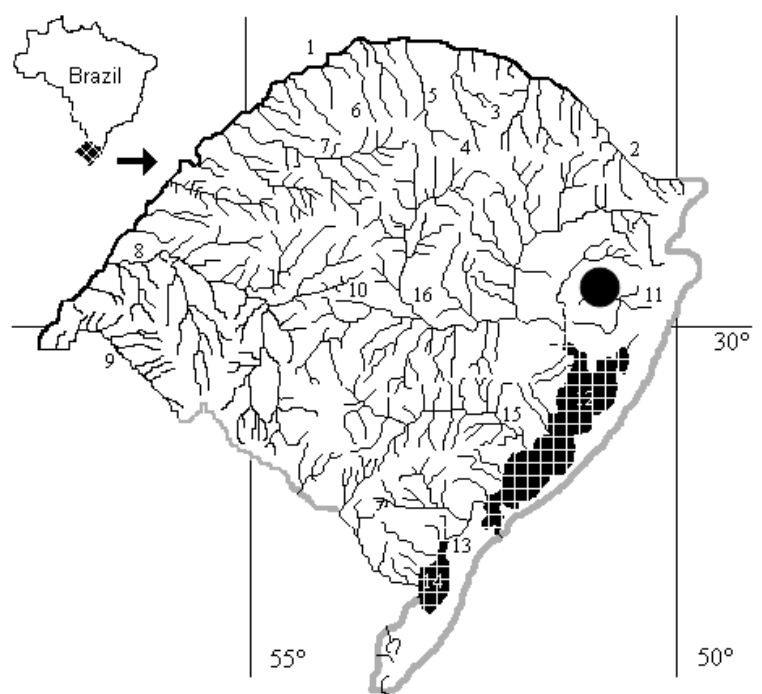

Figura 1. Mapa hidrográfico do estado do Rio Grande do Sul, Brasil No detalhe em preto a região do município de Igrejinha (29³3'10,2'’s; 5050'57'’ W)

se com trechos de declives acentuados, ricos em pequenas cascatas e quedas d'água, além de planícies de corredeiras moderadas ou sem correnteza.

Foi adotada a técnica de amostragem randômica simples (Cochran, 1965). Os indivíduos foram coletados manualmente, com o auxílio de um puçá, ao longo de 60 metros do arroio. O processo de amostragem dos indivíduos consistiu no revolvimento do substrato, sempre no sentido oposto ao da correnteza, de tal maneira que os indivíduos ali presentes fossem levados pela correnteza até o interior do puçá.

Após a captura, os animais foram triados por sexo, medidos quanto ao comprimento do cefalotórax (CC) e devolvidos ao arroio no mesmo local de onde foram retirados. As medições foram realizadas com o auxílio de um paquímetro digital com precisão de $0,01 \mathrm{~mm}$ e uma lupa manual.

Nos indivíduos em que a identificação do sexo não foi possível, estes foram considerados como juvenis e alocados integralmente para ambos os sexos (GONÇALVES et al., 2006).

Os animais com sexo definido, porém menores que a menor fêmea ovígera encontrada em campo (11,04 mm de CC) foram considerados como machos jovens e fêmeas jovens.

O crescimento em comprimento de machos e fêmeas de A. itacolomiensis foi realizado pela análise das distribuições de frequências absolutas do CC em intervalos de classe de $1,0 \mathrm{~mm}$, correspondente a $1 / 4$ do desvio padrão do CC de machos e fêmeas (MARKUs, 1971). O deslocamento das modas dos grupos etários na população foi acompanhado ao longo dos meses de amostragem. As modas dos histogramas de frequências do CC foram calculadas segundo SPIEGEL (1979), conforme a equação:

Moda $=\mathrm{L} 1+(\Delta 1 / \Delta 1+\Delta 2) \cdot \mathrm{C}$, onde: $\mathrm{L} 1=$ limite real inferior da classe modal; $\Delta 1=$ excesso da frequência modal sobre a da classe imediatamente inferior; $\Delta 2=$ excesso da frequência modal sobre a da classe imediatamente superior; $\mathrm{C}=$ amplitude do intervalo da classe modal.

O tamanho médio do CC dos menores animais encontrados em campo, correspondentes a 35 indivíduos juvenis, que se encontravam aderidos aos pleópodos das fêmeas, foi considerado como a primeira moda, tanto para machos quanto para fêmeas.

Os dados foram agrupados trimestralmente considerando-se as estações do ano, onde o inverno foi a primeira estação amostrada. O modelo de crescimento adotado para a determinação das curvas de crescimento de machos e fêmeas foi o de Von BerTalanfFy (1938), seguindo-se a equação:

$\mathrm{Ct}=\mathrm{C} \infty\left[1-\mathrm{e}^{-\mathrm{k}(\mathrm{t}+\mathrm{t} 0)}\right]$, onde: $\mathrm{Ct}=$ comprimento do cefalotórax (mm) na idade $\mathrm{t} ; \mathrm{C} \infty=$ comprimento médio máximo do cefalotórax $(\mathrm{mm}) ; \mathrm{e}=$ base dos logaritmos neperianos; $\mathrm{k}$ $=$ constante relacionada à taxa de crescimento $\mathrm{t}=$ idade dos indivíduos (dias); $\mathrm{t}_{0}=$ parâmetro relacionado com o tamanho do animal ao nascer.

As curvas de crescimento para machos e fêmeas foram ajustadas utilizando-se o método de Ford-Walford (WALFORD, 1946) para amostragens com mesmo intervalo de tempo. Com base nas regressões lineares obtidas foram calculados o $\mathrm{C} \infty$, $\mathrm{k}$ o t , utilizando-se a seguinte equação de acordo com FABENS (1965): (1) $\mathrm{C} \infty=\mathrm{a} / \mathrm{-b}$; (2) $\mathrm{k}=-\mathrm{b}$; $\mathrm{t}_{0}=\mathrm{k}^{-1} \cdot \ln \left\{\Sigma\left[(\mathrm{C} \infty-\mathrm{Ct}) \cdot \mathrm{e}^{(-\mathrm{k} . \mathrm{t})}\right] / \Sigma\left[\left(\mathrm{C} \infty \cdot \mathrm{e}^{-2 \mathrm{kt}}\right)\right]\right\}$, onde "a" e "b" são parâmetros das regressões transformadas pelo método de Ford-Walford (WALFORD, 1946).

As curvas de crescimento de ambos os sexos foram linearizadas segundo o método proposto por ALLEN (1976): $\mathrm{Ct}=\mathrm{a}-\mathrm{b} \cdot \mathrm{r}^{\mathrm{t}}$, onde $\mathrm{a}=\mathrm{C} \infty ; \mathrm{b}=\mathrm{C} \infty . \mathrm{e}^{\mathrm{k} . \mathrm{t}} ; \mathrm{r}=$ médio $=\left(\mathrm{e}^{-\mathrm{k}}\right.$ dos machos $+\mathrm{e}^{-\mathrm{k}}$ das fêmeas $) / 2$. Após esta transformação, as retas obtidas foram comparadas pela análise de covariância (ANCOVA; $\alpha=0,05)$ (ZAR, 1996).

A estimativa do tempo de vida dos animais foi realizada por meio da substituição do parâmetro " $t$ ", da equação calculada para a curva de crescimento de machos e fêmeas pelo número de dias transcorridos entre uma amostragem e outra, no caso 90 dias, conforme o modelo proposto por VON BERTALLANFY (1938).

\section{RESULTADOS E DISCUSSÃO}

Ao longo do período amostral foram analisados 1.278 indivíduos de A. itacolomiensis, sendo 543 machos (314 jovens $(57,83 \%)$ e 229 adultos $(42,17 \%)$ ), 486 fêmeas (255 jovens $(52,47 \%)$ e 231 adultas $(47,53 \%)$ ) e 249 juvenis. A medida do comprimento cefalotorácico (CC) variou de 4,26 a 21,82 $\mathrm{mm}(10,76 \pm 0,15)$ para os machos e de 3,22 a $17,29 \mathrm{~mm}(10,77 \pm 0,12)$ para as fêmeas. Os juvenis mediram entre 1,59 e $7,32 \mathrm{~mm}$ de CC $(4,77 \pm 0,09)$.

As distribuições de frequências absolutas sazonais do CC (mm) para ambos os sexos estão evidenciadas na figura 2. O recrutamento ocorreu durante os meses de outubro, novembro e dezembro, correspondendo às estações primavera e verão.

Nas distribuições de frequência de comprimento do $\mathrm{CC}$ de machos e fêmeas, observa-se a presença de três grupos etários formados por indivíduos juvenis que estão entrando na população ao longo das estações, indivíduos de tamanhos intermediários, oriundos de coortes anteriores aos juvenis e um grupo etário dos animais mais velhos, provavelmente oriundos de recrutamentos do ano anterior aos demais animais e que estariam em seu segundo ano de vida (Fig. 2).

As modas calculadas para cada estação do ano encontram-se na tabela I. 
As curvas de crescimento em comprimento do cefalotórax calculadas através do deslocamento modal para machos e fêmeas de A. itacolomiensis são descritas, respectivamente, pelas seguintes equações: $\mathrm{C}_{\mathrm{t}}=23,21\left[1-\mathrm{e}^{-0,0094(\mathrm{t}+7,03)}\right] \mathrm{e} \mathrm{C}_{\mathrm{t}}=19,49\left[1-\mathrm{e}^{-0,0065(\mathrm{t}+11,16)}\right]$ (Fig. 3).

O tamanho máximo do cefalotórax estimado por meio da curva de crescimento, foi de $23,21 \mathrm{~mm}$ para machos e 19,49 mm para fêmeas. Esses valores aproximamse aos registrados para os maiores animais encontrados em campo, sendo 21,82 mm de CC para machos e 19,29 $\mathrm{mm}$ de CC para fêmeas. A taxa de crescimento dos machos foi superior à das fêmeas $(0,0094$ e 0,0065 respectivamente).
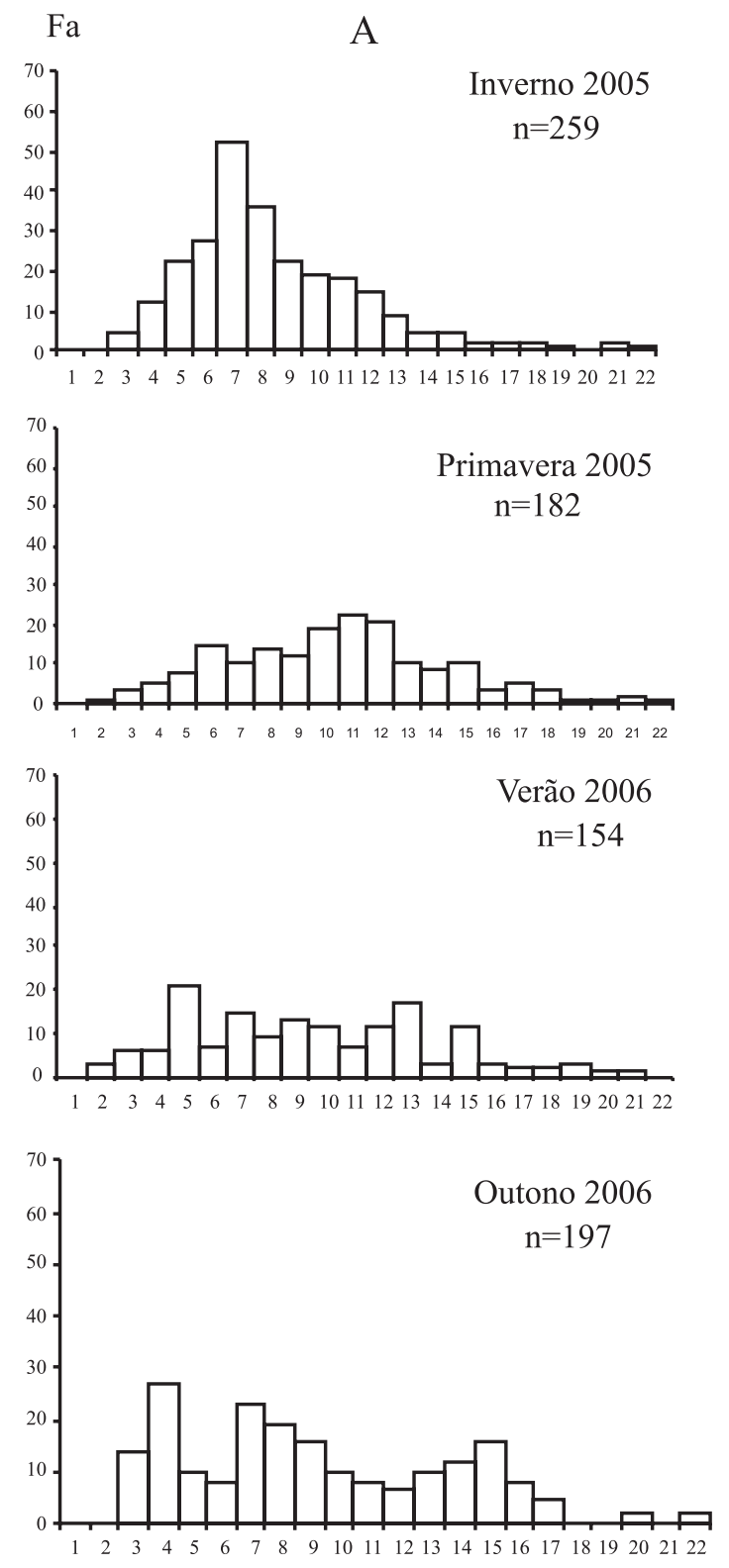

O tempo de vida estimado por intermédio do cálculo da curva de crescimento para A. itacolomiensis foi de 2,2 anos para os machos e 2,5 anos para as fêmeas.

A comparação entre as curvas de crescimento linearizadas de machos e fêmeas, evidenciou que não existe diferença significativa na homogeneidade das variâncias residuais $\left(\mathrm{Fc}=3,2<\mathrm{F}_{0,05, \mathrm{gl} 4,4}\right)$, possibilitando assim a comparação das declividades e das elevações das retas. Observou-se que o valor de $\mathrm{Fc}\left(1.064,11>\mathrm{F}_{0,05}\right.$ g1 4,4 ) foi maior que Ft, concluindo-se que A. itacolomiensis apresenta um crescimento diferenciado entre os sexos, com os machos apresentando um maior crescimento.

A diferença do tamanho assintótico entre os sexos
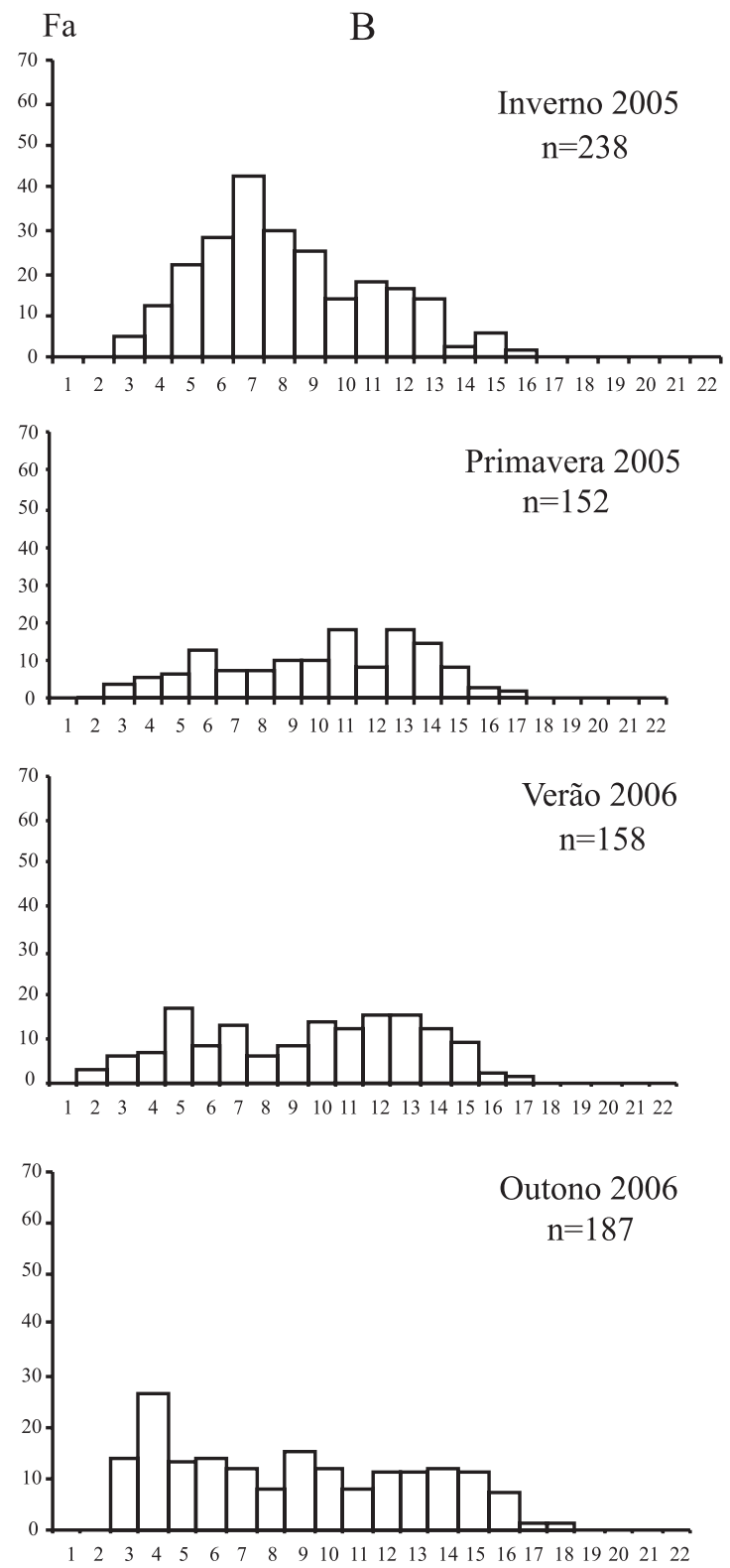

Classes de comprimento do cefalotórax ( $\mathrm{mm}$ )

Figura 2. Distribuição das frequências absolutas sazonais do comprimento do cefalotórax (mm) de machos (A) e fêmeas (B) de Aegla itacolomiensis Bond-Buckup \& Buckup, 1994, amostradas no período de junho de 2005 a maio de 2006 no arroio Solitária Alta, tributário da bacia hidrográfica do rio dos Sinos, RS. $(1)=0 \dashv 1,(2)=1 \dashv 2,(3)=2 \dashv 3,(4)=3 \dashv 4,(5)=4 \dashv 5,(6)=5 \dashv 6,(7)=6 \dashv 7,(8)=7 \dashv 8$, (9) $=8 \dashv 9,(10)=9 \dashv 10,(11)=10 \dashv 11,(12)=11 \dashv 12,(13)=12 \dashv 13,(14)=13 \dashv 14,(15)=14 \dashv 15,(16)=15 \dashv 16,(17)=16 \dashv 17,(18)=17 \dashv 18$, $(19)=18 \dashv 19,(20)=19 \dashv 20,(21)=20 \dashv 21,(22)=21 \dashv 22$. Fa $=$ Frequência absoluta de indivíduos. 
de A. itacolomiensis, onde os machos apresentam-se maiores que as fêmeas, parece ser uma característica comum a quase todas as espécies de eglídeos já estudadas, como em A. leptodactyla, A. jarai e A. longirostri. Uma exceção a essa característica foi constatada por BUENo et al. (2000) em seu estudo sobre o crescimento de $A$. platensis, no qual registraram que as fêmeas apresentavam o tamanho assintótico maior do que os machos (Tab. II). No entanto, os autores atribuíram essa diferença ao fato de que os machos maiores da população foram raros durante as amostragens.
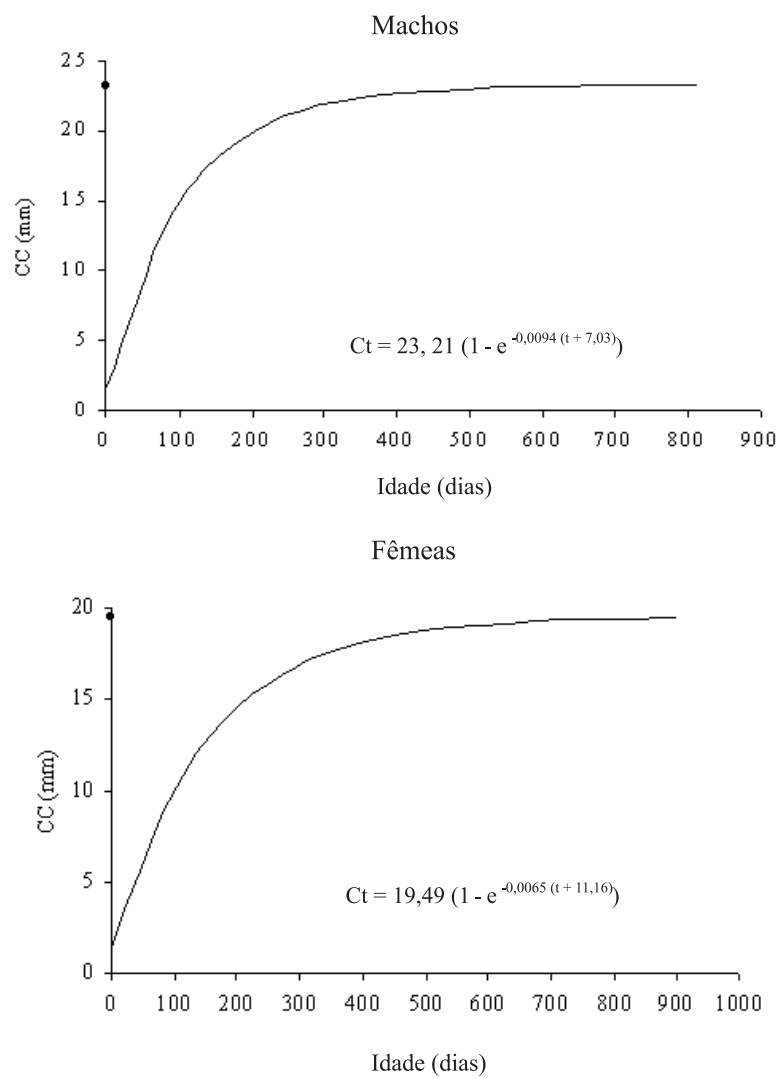

Figura 3. Curvas de crescimento em comprimento do cefalotórax (CC) de machos e fêmeas de Aegla itacolomiensis Bond-Buckup \& Buckup, 1994, amostradas no período de junho de 2005 a maio de 2006 no arroio Solitária Alta, tributário da bacia hidrográfica do rio dos Sinos, RS.

Tabela I. Modas utilizadas para o cálculo das curvas de crescimento de machos e fêmeas de Aegla itacolomiensis Bond-Buckup \& Buckup, 1994.

\begin{tabular}{lcccc}
\hline & Inverno & Primavera & Verão & Outono \\
& 2005 & 2005 & 2006 & 2006 \\
\hline Machos $(\mathrm{mm})$ & 1,23 & 14,14 & 20,00 & 21,50 \\
Fêmeas $(\mathrm{mm})$ & 1,23 & 10,47 & 12,00 & 17,00 \\
\hline
\end{tabular}

O fato dos machos possuírem tamanhos superiores aos das fêmeas pode estar relacionado a taxas diferenciadas de crescimento entre os sexos, de tal forma que os machos apresentam elevada taxa de crescimento em determinado período da sua vida, enquanto as fêmeas direcionam uma importante parcela da sua energia para os eventos da reprodução. Além disso, a diferenciação do crescimento somático entre os sexos pode estar relacionada a comportamentos circadianos distintos entre os indivíduos. A preferência de machos e fêmeas por nichos distintos no meio onde vivem e o deslocamento no ambiente na busca por alimento poderia estar ocorrendo em $A$. itacolomiensis (WARNER, 1967; GIESEL, 1972; Wolf et al., 1975; MAUCHLINE, 1977; Montague, 1980; Díaz \& Conde, 1989).

A taxa de crescimento dos animais pode estar diretamente ligada a fatores abióticos que exercem influência sobre os indivíduos de uma população, podendo inclusive desencadear ou alterar processos fisiológicos relacionados entre outros, aos eventos da reprodução e ao crescimento (DíAz et al., 2003, CREAR et al., 2003). No presente trabalho, registrou-se que os machos de A. itacolomiensis possuem uma taxa de crescimento mais elevada do que as fêmeas. Este resultado é corroborado por outros trabalhos já realizados com as populações de A. platensis, A. jarai e A. longirostri (BuENo et al., 2000; Boss et al., 2006 e SiLvACAstiglioni et al., 2006 respectivamente). No entanto, em estudo realizado com A. leptodactyla (Noro \& BuCKUP, 2003), os autores constataram taxas de crescimento muito similares em ambos os sexos (Tab. II).

Fatores como temperaturas elevadas e períodos prolongados de exposição à luz (fotoperíodo) podem figurar entre os aspectos que condicionam o crescimento dos eglídeos. Variações climáticas nos sistemas límnicos onde esses animais vivem podem influenciar nas suas atividades circadianas, incluindo aquelas dedicadas ao forrageamento, além de afetar a abundância de recursos alimentares nos cursos d'água, o que acaba por refletir no crescimento somático dos organismos (CHIPPs et al., 2000; Kumlu \& Eroldogan, 2000; Crear et al., 2003).

Os locais onde vivem as populações de $A$. itacolomiensis, A. platensis, A. jarai e A. longirostri diferenciam-se significativamente em algumas das suas características abióticas, como altitude e temperatura média anual. Como exemplo, A. leptodactyla. ocorre a uma altitude de $1.200 \mathrm{~m}$, no município de São José dos Ausentes, na região serrana do Estado do Rio Grande do Sul, que apresenta-se sob domínio do clima mesotérmico médio, havendo, pelo menos, um mês com temperatura média inferior a $10^{\circ} \mathrm{C}$. Este clima se caracteriza por possuir um inverno acentuado e verões com temperaturas amenas pelo efeito da altitude (NIMER, 1989). A temperatura média anual, obtida através de dados do IPAGRo (1989) para a região é de $15^{\circ} \mathrm{C}$, a média das máximas do ano é de $21^{\circ} \mathrm{C}$ e a média das mínimas anuais é de $10^{\circ} \mathrm{C}$ (Tab. III). Dessa

Tabela II. Comparação dos valores obtidos para o comprimento assintótico $(\mathrm{C} \infty)$ e para a taxa de crescimento (k) entre espécies de eglídeos.

\begin{tabular}{|c|c|c|c|c|c|}
\hline Espécie & $\mathrm{C} \infty(\mathrm{mm}) \varnothing^{7}$ & $\mathrm{C} \infty(\mathrm{mm}) \stackrel{\text { P }}{\mathrm{N}}$ & $\mathrm{k} \mathrm{O}^{2}$ & $\mathrm{k}$ 우 & Autor \\
\hline A. itacolomiensis & 23,21 & 19,49 & 0,0094 & 0,0065 & Presente trabalho \\
\hline A. leptodactyla & 19,83 & 18,96 & 0,0023 & 0,0024 & NoRo \& Buckup (2003) \\
\hline A. jarai & 25,11 & 23,33 & 0,0082 & 0,0048 & Boss et al. (2006) \\
\hline A. longirostri & 27,9 & 21,45 & 0,0069 & 0,0048 & Silva-Castiglioni et al. (2006) \\
\hline A. platensis & 17,39 & 19,12 & 0,0041 & 0,0033 & BuENo et al. (2000) \\
\hline
\end{tabular}


Tabela III. Temperaturas médias anuais e altitudes registradas para as localidades dos estados do Rio Grande do Sul (RS) e Santa Catarina (SC), Brasil, onde foram realizados trabalhos sobre o crescimento de eglídeos.

\begin{tabular}{|c|c|c|c|c|}
\hline Espécie & Local de coleta & $\begin{array}{l}\text { Temperatura } \\
\text { média anual }\end{array}$ & Altitude média & Autor \\
\hline A. itacolomiensis & Igrejinha, RS & $19,7^{\circ} \mathrm{C}$ & $18 \mathrm{~m}$ & Presente trabalho \\
\hline A. leptodactyla & São José dos Ausentes, RS & $15^{\circ} \mathrm{C}$ & $1.200 \mathrm{~m}$ & NoRo \& BuCKuP (2003) \\
\hline A. jarai & Blumenau, SC & $21,5^{\circ} \mathrm{C}$ & $21 \mathrm{~m}$ & Boss et al. (2006) \\
\hline A. longirostri & Itaára, RS & $20^{\circ} \mathrm{C}$ & $400 \mathrm{~m}$ & Silva-CASTiglioni et al. (2006) \\
\hline A. platensis & Taquara, RS & $23^{\circ} \mathrm{C}$ & $57 \mathrm{~m}$ & BuENO et al. (2000) \\
\hline
\end{tabular}

forma, o fato dos exemplares crescerem de forma mais lenta que as demais espécies do gênero estudadas até o momento, ou seja, apresentarem taxas de crescimento com valores mais baixos (Tab. II) pode estar relacionado à influência desses fatores abióticos.

A estimativa de longevidade para machos e fêmeas de A. itacolomiensis foi semelhante à encontrada para outros eglídeos, como em A. platensis, estimada em 2,5 anos para ambos os sexos (BUENO \& BOND-BUCKUP, 2000). Em A. longirostri e A. jarai os machos vivem em média 1,9 e 2 anos respectivamente e as fêmeas de ambas as espécies cerca de 2 anos (Silva-CASTiglioni et al., $2006 \mathrm{e}$ Boss et al., 2006, respectivamente).

Quando comparados os resultados obtidos no presente trabalho aos de outros estudos de mesmo cunho já realizados com eglídeos, observa-se que o crescimento de A. itacolomiensis, em ambiente natural, assemelha-se mais ao das espécies A. jarai e A. longirostri do que ao da espécie A. leptodactyla. Esta última apresenta uma taxa de crescimento consideravelmente inferior ( $\%$ : $25 \%$ ○: $37 \%$ ) sugerindo que temperaturas médias anuais amenas como as encontradas na região serrana do estado do Rio Grande do $\operatorname{Sul}\left(15^{\circ} \mathrm{C}\right)$ possam influenciar a taxa de crescimento nesses crustáceos. O crescimento até hoje descrito das várias espécies de eglídeos que ocorrem em áreas com fotoperíodo e temperatura semelhantes revela um padrão envolvendo taxas de crescimento semelhantes.

Agradecimentos. Ao Dr. Sandro Santos pela cedência do mapa que ilustra o texto; ao Programa de Pós-Graduação Biologia Animal pelas facilidades no desenvolvimento dos trabalhos de campo e pela concessão da bolsa de mestrado (RG) e ao Conselho Nacional de Desenvolvimento Científico e Tecnológico pela concessão bolsa produtividade (GBB); ao Dr. Célio Magalhães e ao revisor anônimo pela revisão do manuscrito e valiosas sugestões.

\section{REFERÊNCIAS BIBLIOGRÁFICAS}

AlLEN, R. L. 1976. Method for comparing fish growth curves. New Zealand Journal of Marine Freshwater Research 10(Supl. 4):687-692.

Arenas, R. L. 1976. La cordillera de la costa como refúgio de la fauna dulcícola preglacial. Archivos de Biologia y Medicina Experimentales 10:1-40.

Bahamonde, N. \& LóPez, M. T. 1961. Estudios biológicos en la populacion de Aegla laevis laevis (Latreille) de El Monte (Crustacea, Decapoda, Anomura). Investigaciones Zoológicas Chilena 7:19-58.

Bond-Buckup, G. 2003. Família Aeglidae. In: Melo, G. A. S. ed. Manual de Identificação dos Crustacea Decapoda de água doce do Brasil. São Paulo, Loyola. p.21-116.

Bond-Buckup, G. \& Buckup, L. 1994. A Família Aeglidae (Crustacea, Decapoda, Anomura). Arquivos de Zoologia 32(4):159-347.

Boss, H. J; Silva-Castiglioni, D.; Schacht, K.; Buckup, L.; BondBuckup, G. 2006. Crescimento de Aegla jarai Bond-Buckup e
Buckup (Crustacea, Anomura, Aeglidae). Revista Brasileira de Zoologia 23(2):490-496.

Bueno, A. A. P. \& Bond-Buckup, G. 2000. Dinâmica populacional de Aegla platensis Schmitt (Crustacea, Decapoda, Aeglidae). Revista Brasileira de Zoologia 17(1):43-49.

2004. Natural diet of Aegla platensis Schmitt and Aegla ligulata Bond-Buckup \& Buckup (Crustacea, Decapoda, Aeglidae) from Brazil. Acta Limnologica Brasiliensia 16(2): 115-127.

Bueno, A. A. P.; Bond-Buckup, G. \& Buckup, L. 2000. Crescimento de Aegla platensis Schmitt em ambiente natural (Crustacea, Decapoda, Aeglidae). Revista Brasileira de Zoologia 17(1):51-60.

Chipps, S. R.; Einfalt, L. M. \& Wahl, D. H. 2000. Growth and food consumption by Tiger muskellunge: Effects of temperature and ration level on bioenergetic model predictions. American Fisheries Society 129:186-193.

Cochran, W. G. 1965. Técnicas de Amostragem. Rio de Janeiro, Fundo de Cultura. 555p.

Crear, B. J.; Hart, P. R. \& Thomas, C. W. 2003. The effect of photoperiod on growth, survival, colour and activity of juvenile southern rock lobster, Jasus edwadsii. Aquaculture Research 34:439-444.

Díaz, H. \& Conde, J. E. 1989. Population dynamics and life of mangrove crab Aratus pisonii (Brachyura, Grapsidae) in a marine environment. Bolletin of Marine Science 45(1): 148-163.

Díaz, A. C.; Sousa, L. G.; Cunartas, E. I. \& Petriella, A. M. 2003. Growth, molt and survival of Palaemonetes argentinus (Decapoda, Caridea) under different light-dark conditions. Iheringia, Série Zoologia, 93(3):249-254.

Esteves, F. A. 1988. Fundamentos de Limnologia. Rio de Janeiro, Interciência. 575p.

FABENS, A. J. 1965. Properties and fitting of the von Bertalanffy growth curve. Growth (29):265-289.

Giesel, J. T. 1972. Sex ratio, rate of evolution, and environmental heterogeneity. American Naturalist 106:380-387.

Gonçalves, R. S.; Castiglioni, D. S. \& Bond-Buckup, G. 2006. Ecologia populacional de Aegla franciscana (Crustacea, Decapoda, Anomura) em São Francisco de Paula, RS, Brasil. Iheringia, Série Zoologia, 96:109-114.

Ipagro. 1989. Atlas Agroclimático do Estado do Rio Grande do Sul. Governo do Estado do Rio Grande do Sul, Secretaria da Agricultura e abastecimento, Departamento de Pesquisa, Instituto de pesquisas Agronômicas, Seção de Ecologia Agricola. v.3, p.211-326.

Kumlu, M. \& Eroldogan, T. 2000. Effects of temperature and substrate on growth and survival of Penaeus semisulcatus (Decapoda: Penaeidae) postlarvae. Turkish Journal of Zoology 24:337:341.

López, M. T. 1965. Estudios biológicos em Aegla odebrechtii paulensis Schmitt (Crustacea, Decapoda, Anomura). Boletim de Zoologia da Faculdade de Filosofia, Ciências e Letras da USP 25:301-314.

Markus, R. 1971. Elementos de estatística aplicada. Porto Alegre, Faculdade de Agronomia e Veterinária da UFRGS, Centro Acadêmico Leopoldo Cortez. 329p.

Mauchline, J. 1977. Growth of shrimps, crabs and lobsters: An assessment. Journal du Conseil International Exploration de la Mer 37(2):162-169.

Montague, C. L. 1980. A natural history of temperate western Atlantic fiddler crabs (Genus Uca) with reference to their impact on the salt marsh. Contributions of Marine Science 23:25-55. 
Nimer, E. 1989. Climatologia do Brasil. Rio de Janeiro, IBGE. $421 \mathrm{p}$.

Noro, C. K. \& Buckup, L. 2003. O crescimento de Aegla leptodactyla Buckup \& Rossi, 1977 (Crustacea, Anomura, Aeglidae). Revista Brasileira de Zoologia 20(2):169-182.

Rodriguez, W. \& Hebling, N. J. 1978. Estudos biológicos em Aegla perobae Hebling \& Rodriguez, 1977 (Decapoda, Anomura). Revista Brasileira de Biologia 38(2):383-390.

Silva-Castiglioni, C.; Barcelos, D. F. \& Santos, S. 2006. Crescimento de Aegla longirostri Bond-Buckup \& Buckup (Crustacea, Anomura, Aeglidae). Revista Brasileira de Zoologia 23(2):408-413.

Spiegel, M. R. 1979. Estatística. São Paulo, McGraw-Hill. 580p. Swiech-Ayoub, B. P. \& Masunari, S. 2001. Flutuações temporal e espacial de abundância e composição de tamanho de Aegla castro Schmitt (Crustacea, Anomura, Aeglidae) no Buraco do Padre, Ponta Grossa, Paraná, Brasil. Revista Brasileira de Zoologia 18(3):1003-1017.
Vaz-Fereira, R.; Gray, R. \& Vaz-Ferreira, M. 1945. Notas biométricas sobre crustáceos decápodos del Gênero Aegla Leach, I La variación de algunas magnitudes en Aegla uruguayana Schmitt. Comunicaciones Zoologicas Del Museo De Historia Natural De Montevideo 1(24):1-6.

Von Bertalanffy, L. 1938. A quantitative theory of organic growth (inquiries on growth laws II). Human Biology 10(1):181-213.

Walford, L. A. 1946. A new graphic method of describing the growth of animals. Biological Bulletin 90(2):141147.

Warner, G. F. 1967. The life history of the mangrove tree crab Aratus pisoni. Journal of Zoology 153:321-335.

Wolf, P.; Shanholtaer, S. F. \& Reimold, R. J. 1975. Population estimates for Uca pugnax on Duplin estuary marsh. Crustaceana 29:79-91.

$Z_{\mathrm{AR}}$, J, H. 1996. Biostatistical analysis. Upper Sadle River, Prentice-Hall. 662p. 